This item was submitted to Loughborough's Institutional Repository (https://dspace.lboro.ac.uk/) by the author and is made available under the following Creative Commons Licence conditions.

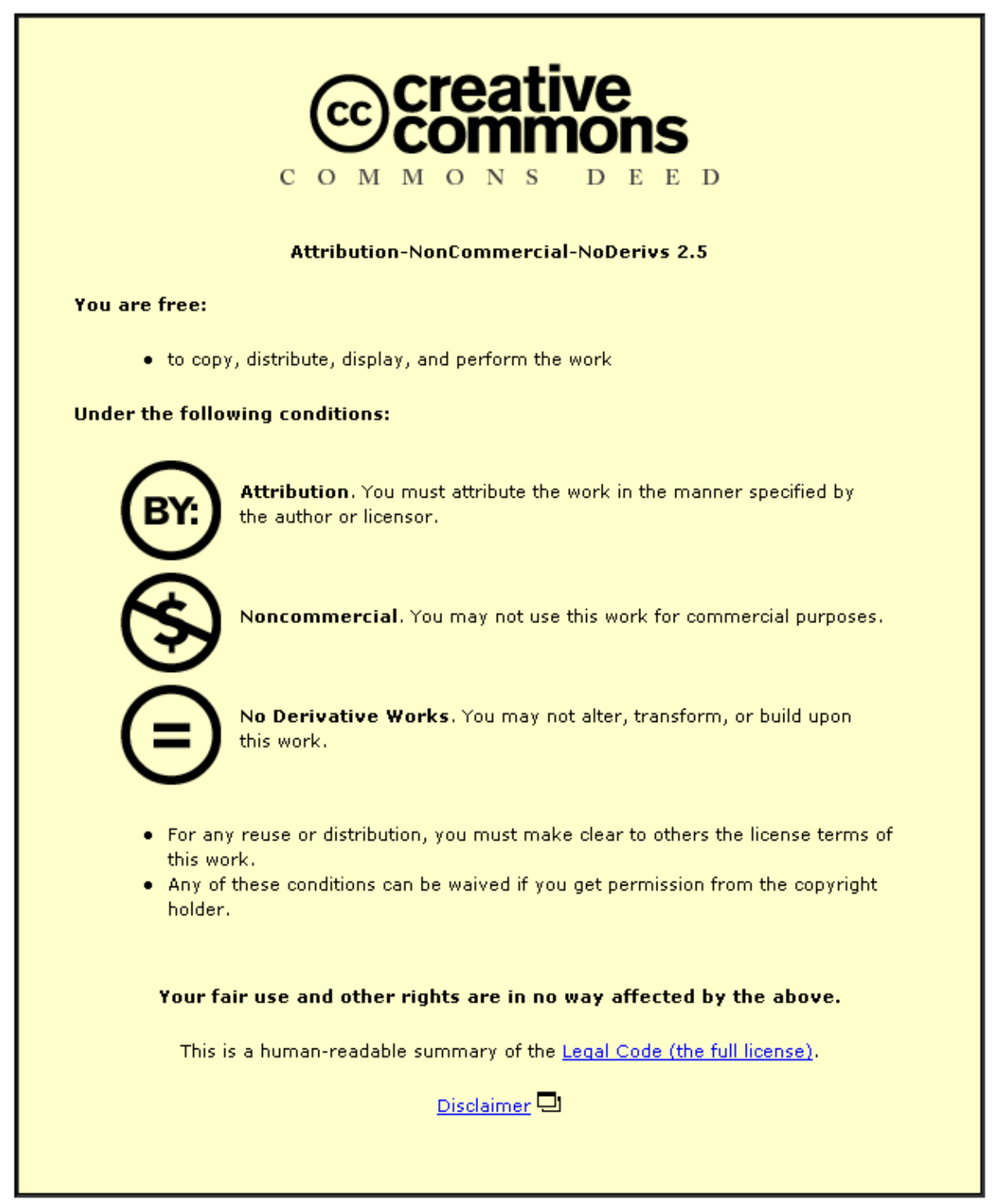

For the full text of this licence, please go to: http://creativecommons.org/licenses/by-nc-nd/2.5/ 


\title{
Microflotation Performance for Algal Separation
}

James Hanotuํㅜ HC Hemaka Bandulasena1 William B Zimmerman ${ }^{1 *}$

${ }^{1}$ Department of Chemical and Biological Engineering, University of Sheffield, Mappin Street, Sheffield S1 3JD, United Kingdom

*Corresponding author. Email: w.zimmerman@shef.ac.uk

\begin{abstract}
The performance of microflotation, dispersed air flotation with microbubble clouds with bubble size about 50 microns, for algae separation using fluidic oscillation for microbubble generation is investigated. This fluidic oscillator converts continuous air supply into oscillatory flow with a regular frequency to generate bubbles of the scale of the exit pore. Bubble characterisation results showed that average bubble size generated under oscillatory air flow state was $86 \mu \mathrm{m}, \sim$ twice the size of the diffuser pore size of $38 \mu \mathrm{m}$. In constrast, continuous air flow at the same rate through the same diffusers yielded an average bubble size of $1059 \mu \mathrm{m}, 28$ times larger than the pore size. Following microbubble generation, the separation of algal cells under fluidic oscillator generated microbubbles was investigated by varying metallic coagulant types, concentration and $\mathrm{pH}$. Best performances were recorded at the highest coagulant dose $(150 \mathrm{mg} / \mathrm{L})$ applied under acidic conditions ( $\mathrm{pH} \mathrm{5})$. Amongst the three metallic coagulants studied, ferric chloride yielded the overall best result of $99.2 \%$ under the optimum conditions followed closely by ferric sulphate (98.1\%) and aluminium sulphate with $95.2 \%$. This compares well with conventional dissolved air flotation (DAF) benchmarks which has a highly turbulent flow, whereas microflotation is laminar with several orders of magnitude lower energy density.
\end{abstract}

Keywords: Microflotation, Fluidic Oscillator, Bioflocculation, Dissolved Air Flotation (DAF), Dispersed Air Flotation

\subsection{Introduction}

Flotation has become the mainstay for colloidal particle separation from an aqueous solution. In essence, the key subprocess is the generation of microbubbles that attach to hydrophobic particles, resulting in buoyant aggregates which then rise to the surface of the flotation cell, where following bubble rupture, the particles are recovered(Dai et al., 2000). Recovery of valuable end-products has been the centre of attraction in flotation separation. A large body of experimental evidence show the reclamation of products such as oil (Al-Shamrani et al., 2002b, Al-Shamrani et al., 2002a, Hosny, 1996, Li et al., 2007, Zouboulis and Avranas, 2000), minerals (Englert et al., 2009), algae (Teixeira and Rosa, 2006, Teixeira et al., 2010) and in cases where water scarcity is the challenge, potable water (Kitchener and Gochin, 1981, Edzwald, 1995) can be achieved by flotation separation.

Algae, in particular, are a reasonable target for flotation separations for biomass processing, but as yet untried with the dense solutions produced from algal cultivation. 
Pienkos and Darzins (2009) highlight harvesting and dewatering operations as a key challenge for economic algal biofuels processing. The density can reach $10 \mathrm{~g} / \mathrm{L}$ of dry biomass, which is substantially higher than DAF removal of fine particles in water purification. Gudin and Thepenier (1989) estimated that harvesting can account for 20$30 \%$ of the total production cost. Molina et al. (2003) present possibly the closest technique to microflotation for algal harvesting - flocculation and bioflocculation followed by sedimentation. Flotation is often viewed as "inverted" sedimentation. The Jameson Cell (Yan and Jameson, 2004) is an induced air flotation process which also achieves high separation performance for microalgae (98\%) and phosphorus. When present in effluent water, algae could be a pernicious contaminant in portable water treatment otherwise, but could be regarded as a raw material given the numerous products obtainable from the unicellular organism such as $\beta$-carotene (Borowitzka, 1992) glycerol, biomass and in particular, biofuel from lipid (Chisti, 2007). While most previous works have focused on the production of biomass from algae (Zimmerman et al., 2011b), only few researchers have been concerned with harvesting biomass and lipid from algae. Whether it is for potable water treatment or recovery of algae for biofuel, flotation separation is a viable means for harvesting algae.

However for flotation to be successful, it is vital for particles to be hydrophobic (Gochin and Solari, 1983) and ultimately attach to gas bubbles. Chemical coagulation is employed to aid this process. Through the suppression of the electrical double layer of particles, particle-particle interaction is facilitated, leading to the formation of larger colloidal structures or flocs. Following collision particles adhere to the surface of gas bubbles forming a strong stable particle-bubble union (Dai et al., 2000). Removal of flocs is hugely dependent on the coagulation $\mathrm{pH}$ but another important factor is the bubble size and flux. Bubbles enhance particle recovery by providing the lifting force necessary for transport and separation. Separation efficiency varies inversely with bubble size (Dai et al., 1998, Dai et al., 2000).

Application of gas bubbles in liquid is gaining extensive application across many fields. Generally, these processes entail efficient ways of facilitating bubble-particle interaction in the liquid rather than merely passing the bubbles through the liquid without it actually adhering and lifting the particles out of solution. Best practices however, require that the particles in the aqueous solution attain optimum collision, attachment 
and stability efficiencies respectively (Derjaguin and Dukhin, 1993) with the gas bubble for complete capture prior to reaching the liquid surface. As such, one of the most efficient ways of achieving this is miniaturising the bubbles. Due largely to their high surface area to volume ratio, particle flotation by small bubbles occurs more rapidly and efficiently. Ahmed and Jameson (1985) estimate a 100-fold enhancement in separation performance for fine particles with bubble size reduction from approximately 700 to 70 microns. Further, small bubbles have gentle convective force relative to large bubbles by reason of their low rise velocity (Schulze, 1992), resulting in tender contact with fragile flocs. To this end, several microbubble generation techniques have been thus developed for flotation applications. Examples include: turbulent microflotation (Miettinen et al., 2010), Induced Air Flotation (IAF) (El-Kayar et al., 1993), Dissolved Air flotation (Edzwald, 2010), and Electroflotation (Hosny, 1996). Of the several techniques available, dissolved air flotation and dispersed air flotation are the most widely developed. Specialist microbubble separations have been achieved in minerals processing with colloidal gas aphrons, which are charged microbubbles (Cilliers and Bradshaw, 1996; Waters et al. 2008).

\subsection{Dissolved Air Flotation}

Dissolved air flotation in particular is the most efficient and widely employed flotation option. According to Henry's law, the process essentially requires dissolving air in water at very high pressure. By so doing, the solution becomes supersaturated; leading to nucleation of microbubbles as soon as pressure is reduces at the nozzle. Unfortunately, this process is energy intensive, due to the high pressure required for air dissolution in water as well as the work done by the pump in feeding the saturator with clarified water. Nonetheless, these two energy intensive sections can be completely bypassed with microflotation.

\subsection{Dispersed Air Flotation}

Traditional dispersed air flotation, basically involves the supply of continuous air stream directly into a porous material (usually a nozzle or a diffuser) from where bubbles are generated. By comparison with other microbubble generation methods, this technique is less energy consumptive. However, the natural problem associated with this method is the difficulty in small bubble production. Though it may seem that production only entails reduction in pore size of bubble diffusers, but even producing 
smaller apertures requires absolute care and precision. Reducing diffuser pore size is obviously expensive and demands more expertise compared to larger size production. Also, because more friction arises with fine apertures and through the passages leading to these apertures, more pressure drop is needed. Apart from the challenge with producing fine apertures, (a more overriding factor) another major problem associated with microbubble generation is the detachment of bubbles from the diffuser. Due to the energy that the bubble must overcome prior to detachment, bubbles naturally grow larger until their buoyant force and force exerted by air jet is greater than the force binding them to the diffuser. By supplying more gas, bubble size as well as buoyant force is increased, leading to detachment. However, this stage in traditional dispersed air flotation is more energy consuming as more energy is required through increased gas supply to disengage the bubble from diffuser nozzle (Zimmerman et al., 2008).

\subsubsection{Microflotation}

Engineering breakthroughs that offer robust generation of microbubbles through microfluidic strategies have been very useful. One such breakthrough is the fluidic oscillator for microbubble generation (Zimmerman et al., 2009, Zimmerman et al., 2008, Zimmerman et al., 2011a, Tesař and Bandalusena, 2011). This bistable valve when fitted to a diffuser ensures production of minute bubbles approximately 10 times smaller than those of conventional dispersed air flotation methods with significant savings on energy consumption (Zimmerman et al., 2008). And so, for fine apertures, fine bubbles almost the size of their exit nozzles can be generated using the fluidic oscillator, consuming 2-3 orders of magnitude less energy density than dissolved air flotation (DAF) and traditional dispersed air flotation, with a similar level of capital cost reduction. Microflotation is application of dispersed air flotation with fluidic oscillator generated microbubbles.

This study aims to investigate the performance of microflotation in algal recovery while also considering the effects of $\mathrm{pH}$ and coagulant dose on recovery efficiency. Traditionally, jar tests or zeta potential metering would be used to identify the level of flocculant, coagulant and $\mathrm{pH}$ for optimal flocculation and coagulation. Here, we have varied all these parameters systematically, as the hypothesis that zeta potential neutrality yields best separation performance. Miettinen et al. (2010) explore the limits of fine particle flotation separations, whilst Jiang et al. (2010) demonstrate "contactless flotation" where the traditional electrokinetics of particle agglomeration (Zimmerman, 
2011) and hydrodynamic collection efficiency (Grammatika and Zimmerman, 2001) are not observed, yet flotation is achieved. Because microflotation is much less energetic than other flotation processes, flocculation and coagulation do not occur under turbulent conditions, for which modelling has identified that there is a finite probability of re-dispersal of flocs already formed (Pyke et al., 2003). Microflotation has no random turbulent shear forces to fight against itself.

\subsection{Materials and Method}

\subsection{System Calibration}

In estimating the concentration of algal cells in the flotation column a calibration curve was first obtained using known algae quantities. Five samples of the grown algae were taken at varying concentrations to assay chlorophyll content. Determination of Chlorophyll content of Dunaliella salina cells was done by taking $2 \times 5 \mathrm{ml}$ samples from each algal flask and centrifuging for $10 \mathrm{mins}$. Sample supernatant was quickly discarded and sample resuspended in $1 \mathrm{ml}$ of distilled water before whirl-mixing. Next $4 \mathrm{ml}$ of acetone was added and whirl-mixed again before samples were allowed to stand for 5 mins away from direct sunlight. After 5 mins, samples were centrifuged for $5 \mathrm{mins}$ at $3000 \mathrm{rpm}$ until algal pellet was white. Finally, the green supernatant was transferred to a glass cuvette and OD measured at $645 \mathrm{~nm}$ and $663 \mathrm{~nm}$ against an acetone blank. Estimation of chlorophyll content was achieved using the following equation to give $\mu \mathrm{g}$ chlorophyll ml-1:

$$
\begin{gathered}
\mathrm{OD}_{645} \times 202=\mathrm{y} \\
\mathrm{OD}_{663} \times 80.2=\mathrm{z} \\
\left(\frac{y+z}{2}\right) / 5 m l
\end{gathered}
$$

The result from the assay was then used in calibrating the spectrophotometer (DR 2800 (HACH Lange)) at both wavelengths by establishing a correlation between the algal chlorophyll content and absorbance at $645 \mathrm{~nm}$ and $663 \mathrm{~nm}$.

\section{$2.2 \quad$ Flotation and Recovery}

A schematic representation of the bench scale dispersed air flotation unit is shown in Figure 1. The main rig components comprises: a flotation cell, microbubble generator (fluidic oscillator and $40 \mathrm{~mm}$ stainless steel baffle distributor diffuser). The fluidic 
oscillator (Tesar et al. 2006,(Tesař and Bandalusena, 2011) measures: $10 \mathrm{~cm} \mathrm{x} 5 \mathrm{~cm} \mathrm{x}$ $5 \mathrm{~cm}$ in length, height and width respectively while the flotation unit measures: $50 \mathrm{~cm}$ by $9 \mathrm{~cm}$ in height and diameter respectively. The tests were conducted with the diffuser placed at the bottom of the flotation unit. Three inorganic metallic coagulants used were aluminium sulphate, ferric III chloride and ferric Sulphate (Sigma Aldrich, UK), while hydrochloric acid and sodium hydroxide (Sigma Aldrich, UK) were used for $\mathrm{pH}$ adjustment. The tests were conducted across five (5) pH ranges and five (5) coagulant concentrations.

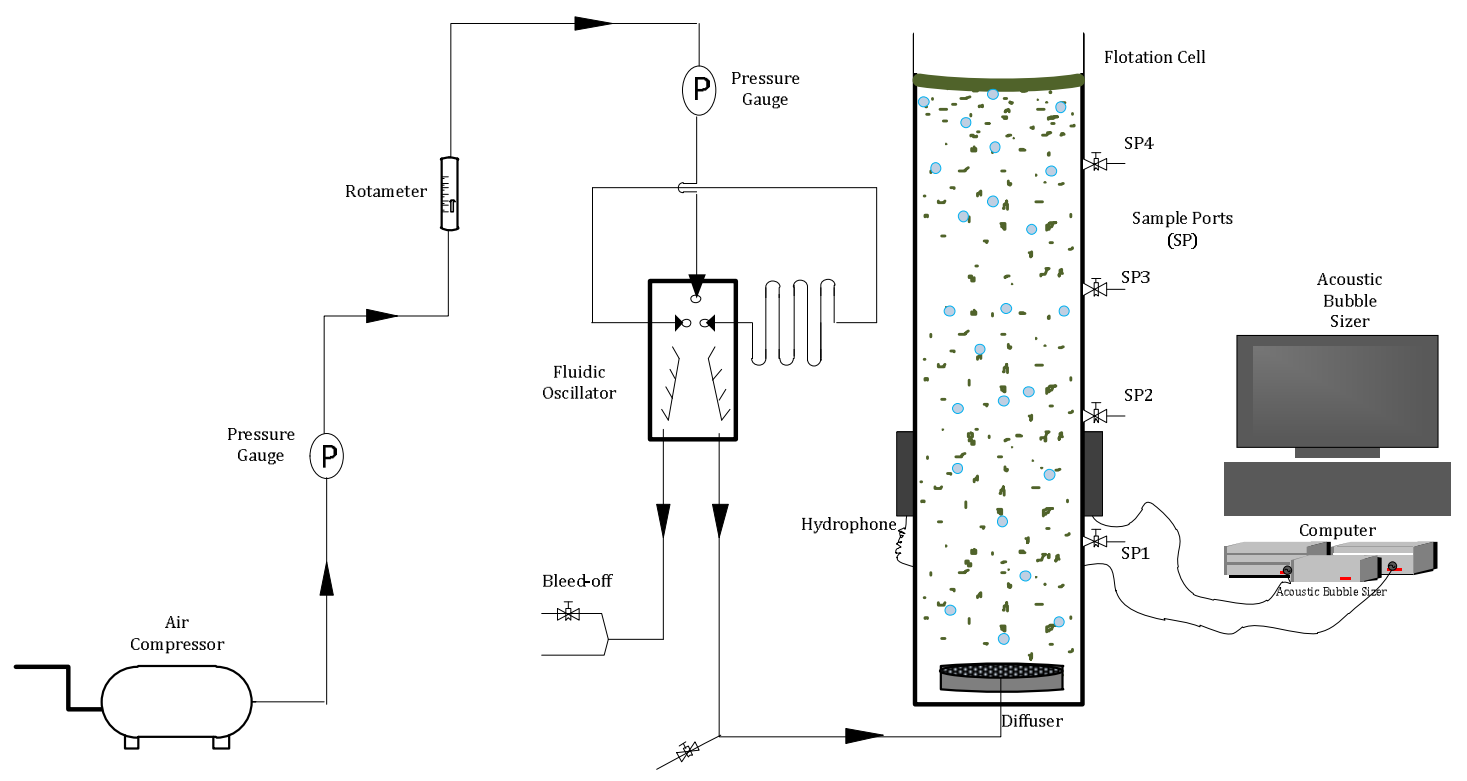

Figure 1: Schematic representation of the experimental set-up. Microfiltered compressed air is fed into the oscillator which then feeds the microporous diffuser with a portion of the air bleed-off or channelled otherwise to another set of diffuser. In this study, a portion of the air was bled off downstream of the fluidic oscillator.

Dunaliella salina 19/30 obtained from the Culture Centre of Algae and Protozoa (CCAP), Oban, Scotland, was previously pre-cultured in a $250 \mathrm{~L}$ airlift Loop Bioreactor containing $248 \mathrm{~L}$ of Dunaliella salina growth medium (Zimmerman et al., 2011c) for 2 weeks. Following that, the microalgae from the laboratory scale $250 \mathrm{~L}$ airlift loop bioreactor was transferred to an outdoor 2200 liter ALB for field trials at Scunthorpe, UK. The microalgae was grown with waste $\mathrm{CO}_{2}$ from steel plant exhaust gas. After $\sim 17$ days, the cultured microalgae from the ALB was emptied into several drums and delivered back to the laboratory for harvesting. Several months after growth the 
microalgae culture was taken for further processing. A two litre microalgae sample at room temperature $\left(20^{\circ} \mathrm{C}\right)$ was mixed to break lumps and disperse the cells homogenously in solution following sedimentation and clustering of cells as a result of prolonged storage. Coagulation and flocculation followed for $4 \mathrm{mins}$ and $10 \mathrm{mins}$ respectively following $\mathrm{pH}$ adjustment. Immediately after flocculating with a mechanical stirrer at 70rpm, the broth was gradually introduced into the flotation column to a height of $30 \mathrm{~cm}$ above diffuser before the microbubble generator was turned on. The diffuser used in this study was made of Perspex material and measures $40 \mathrm{~mm}$ in diameter and overlaid with a stainless steel mesh (Plastok, UK) with pore size of $38 \mu \mathrm{m}$ and an open area of 36\%. Broth samples were collected every three (3) minutes and measured with the calibrated spectrophotometer DR 2800 (HACH Lange) to assay absorbance at 663 and $640 \mathrm{~nm}$ wavelength. Recovery efficiency (R) was determined using the formulae:

$$
R=\left(\frac{C_{i}-C_{f}}{C_{i}}\right)_{100}
$$

where $C_{i}$ and $C_{f}$ are the initial and final algae concentrations respectively.

\subsection{Bubble size Distribution Measurement}

There are two main methods for measuring the size of bubbles generated in a liquid, namely optical and acoustical techniques. Bubble size characterisation using optical means is by far the most widely employed technique. Often times depending on the size and number of bubbles in question as well as the quality of optical device, the optical method can be both painstaking and time consuming to undertake and its accuracy is a function of factors such as light and medium clarity as well as the software for bubble analyses. These factors if not properly addressed can give rise to errors such as underpredicting or over-predicting the bubble diameter particularly in high bubble flux conditions and turbid media.

The acoustic bubble sizer (Dynaflow, Inc.) was developed to meet these challenges while being non-intrusive. By exploiting the ability of bubbles to affect acoustic propagated waves; bubble size and population can be extracted at varying frequencies (Wu and Chahine, 2010). The device consists of a pair of transducer hydrophones made 
of piezoelectric materials inserted in a polyurethane material to prevent contact with water. Both hydrophones are connected to a computer via a control box (see Figure 1). The transmitting hydrophone generates short bursts of sound signals within a set frequency which are then received after travelling through the liquid, by the second hydrophone. The signals are then analysed by special in-built software for processing the phase velocity and attenuation within the desired frequency range to estimate the size distribution of bubbles. The acoustic bubble sizer (ABS) was used in this study for bubbles characterisation. Two sets of flat hydrophone used (measuring: $7.5 \times 7.5 \times 2.5 \mathrm{~cm}$, optimal operating frequency range from $70 \sim 200 \mathrm{kHz}$ and corresponding bubble size of 34-100 $\mu \mathrm{m}$ ) were mounted vertically ( $9 \mathrm{~cm}$ apart) on either side of the flotation column. Three (3) runs were undertaken to determine bubble size distribution under oscillatory condition. The experimental set-up is shown in Figure 1, while the bubble images are displayed in figure 2 and characterisation results are shown in Figures 3 and 4 respectively.

\subsection{Result and Discussion}

\subsection{Bubble Characterisation}

\subsubsection{Bubble Size Distribution}

Microbubble generation is an essential part of flotation separation. The size and number of bubbles is a vital operating and control variable (Edzwald, 2010) and must be appropriate for effective bubble-particle contact. Characterising the bubbles generated from a flotation unit is a necessary first step and was undertaken prior to recovery of algae cells. Photographs of bubbles generated under steady and oscillatory flow states are shown in Figure 2 and in Figure 3, bubble size distribution from the stainless steel mesh diffuser used in the experiment are presented. Under steady air flow (Figure 3a) bubbles produced are several folds larger than the exit aperture. Conversely, Figure 3 (b) shows the mist of microbubbles produced from the same diffuser under oscillatory air flow. 
(a)

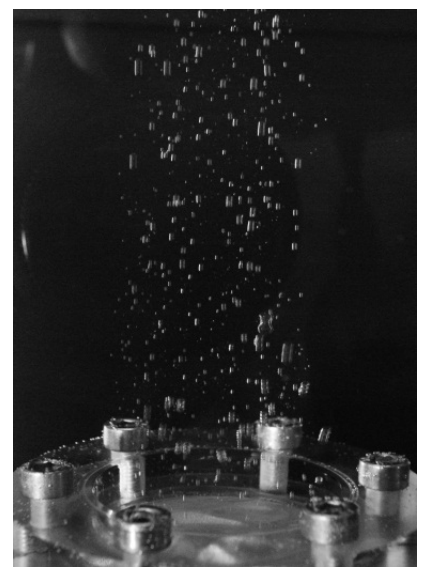

(b)

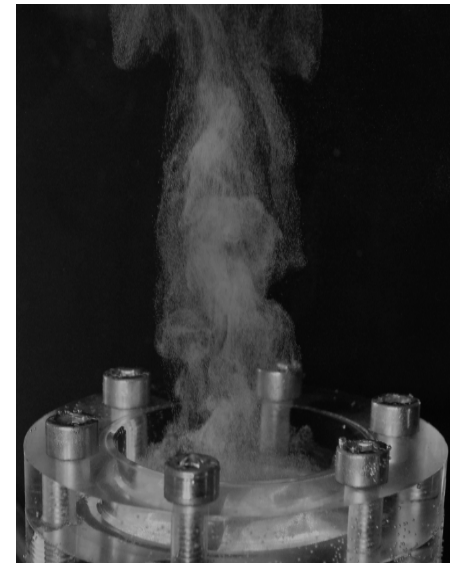

Figure 2 Images of bubbles generated from the same microporous diffuser under different conditions. (a) Bubbles generated under steady air flow. Bubbles are coalescent, non-uniform and several folds larger than the diffuser pore size. (b) Bubble generated under oscillatory flow. Formation of uniformly sized non-coalescent mist of microbubbles almost same size as diffuser pores.

Figure 3 presents the distribution of bubble size generated under steady and oscillated air supply conditions. Under steady flow state (Fig. 3a), two peaks are apparent which is evident of a wide range in bubble size distribution. The highest peaks reveal the dominance of bubbles equal to $650 \mu \mathrm{m}$ and $1350 \mu \mathrm{m}$ respectively. The smallest bubble produced however was $357 \mu \mathrm{m}$ while the largest size measured was $1673 \mu \mathrm{m}$. Average bubble radius recorded was $1059 \mu \mathrm{m}$ with $60 \%$ of the bubbles less than $1287 \mu \mathrm{m}$. From Fig. $3 \mathrm{~b}$, the single peak graph shows a positive skew of bubble size distribution which reveals the dominance of $24 \mu \mathrm{m}$ sized bubbles. The smallest bubble produced was $24 \mu \mathrm{m}$ while the largest size measured was $260 \mu \mathrm{m}$. However, average bubble radius was $86 \mu \mathrm{m}$ with $60 \%$ of the bubbles approximately $74 \mu \mathrm{m}$.

While the difference between the average bubble size under steady and oscillatory air flow conditions is $967 \mu \mathrm{m}$, equally remarkable is that the average bubble size generated with the fluidic oscillator is approximately twice larger than the diffuser pore size $(38 \mu \mathrm{m})$. By contrast, without the oscillator, the average bubble size achieved is several orders of magnitude (28 times) larger than the diffuser pore size. 
(a)

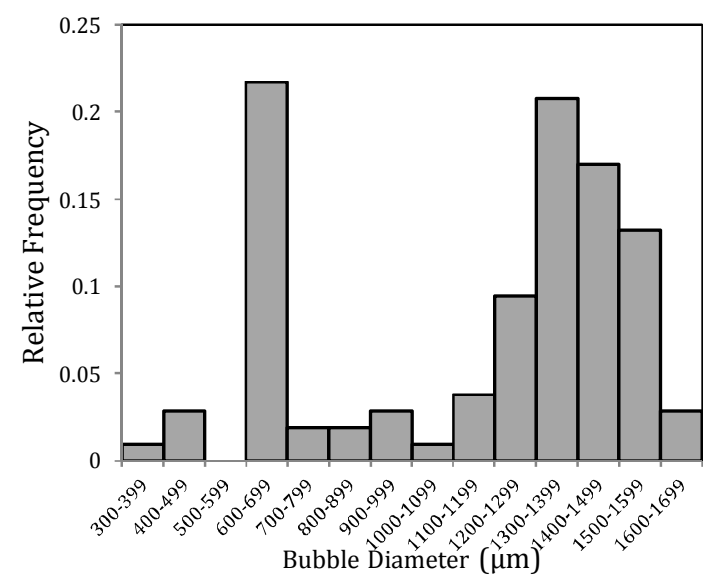

(b)

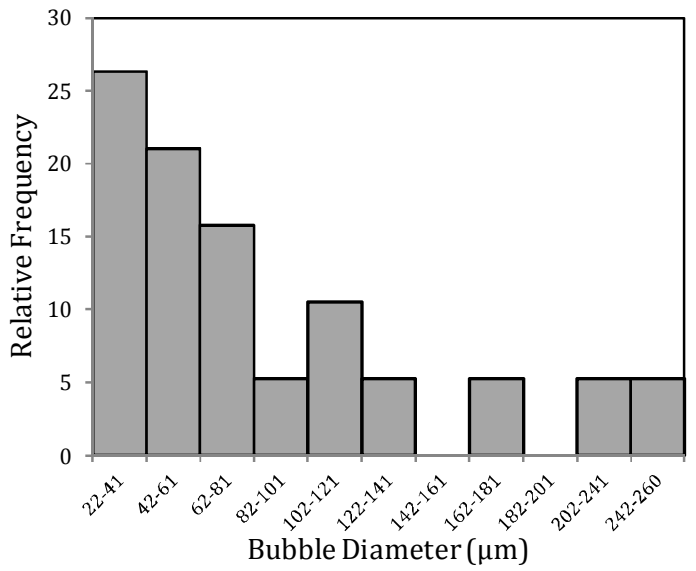

Figure 3: Bubble size distribution graph from the stainless steel mesh diffuser. (a) Graph of bubble size distribution under steady air supply. The bubble sizes were analysed with a high speed camera at 1000 fps and 1024 x 1024 resolution. Minimum and maximum sizes recorded were $181 \mu \mathrm{m}$ and $1673 \mu \mathrm{m}$ respectively with an average bubble size of $1059 \mu \mathrm{m}$. (b) The bubble sizes were analysed with the acoustic bubble sizer. The graph shows distribution of bubbles produced at supply flowrate of $85 \mathrm{~L} / \mathrm{min}$ through the oscillator from the $38 \mu \mathrm{m}$ pore-sized stainless steel membrane diffuser at operating pressure of $\sim 1$ bar. A portion of the air supply downstream the oscillator was bled-off to match diffuser capacity and the bubble size distribution analysed with the acoustic bubble sizer. Average bubble size measured with the $\mathrm{ABS}$ is $86 \mu \mathrm{m}$ with maximum and minimum bubble sizes recorded to be $\sim 24$ and $260 \mu \mathrm{m}$ respectively and standard deviation of $60 \mu \mathrm{m}$.

\subsubsection{Bubble Density Analyses}

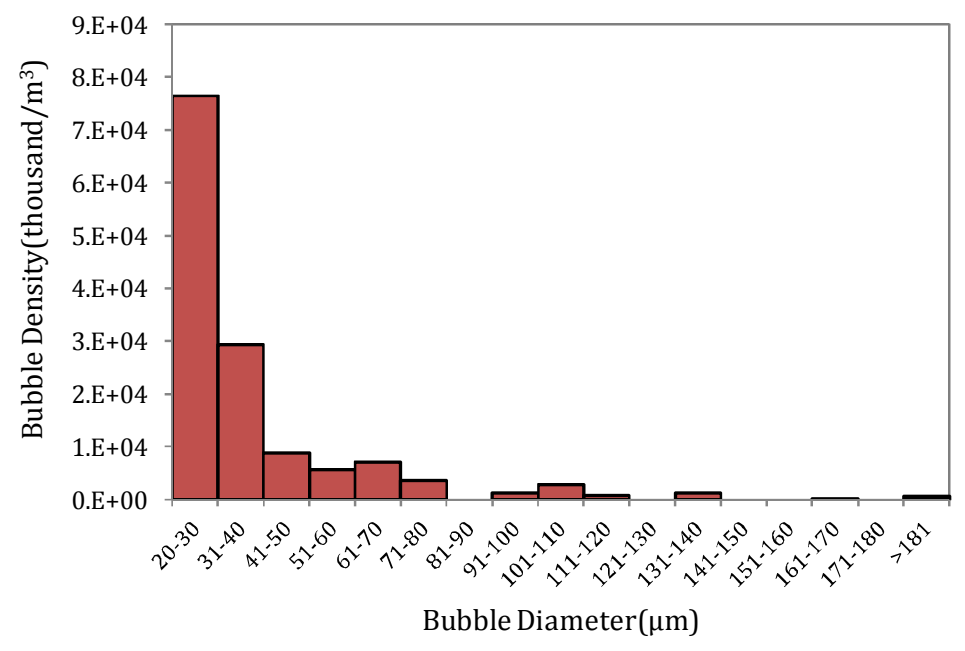

Figure 4: Bubble density from the stainless steel mesh diffuser showing the number of bubbles per unit volume. Microbubbles ranging from 24-40 microns in diameter dominate the flux measured by acoustic bubble spectrometer. 
Another perspective in bubble characterisation considered is the number of bubbles per unit volume for microbubbles generated under oscillatory flow. The bubble density graph presented in figure 4 was determined by measuring the population of bubbles in the column and result showed that 20-40 micron sized bubble made up 95\% of the total bubble density, while $5 \%$ comprised of bubbles greater than 40 microns in a bubble size distribution of 20-260 microns (see figure $3 \mathrm{~b}$ for distribution by size). The narrow range of bubbles size distribution not only strongly suggests the production of largely non-coalescent but more particularly, relatively uniformly sized microbubbles.

The difference in bubble size is simply attributable to the fluidic oscillator. The bistable device facilitates microbubble production by oscillating a stream of continuous air supply. The pulse generated due to the oscillation helps to knock-off bubbles at the developmental stage. In constrast, bubbles continue to grow under continuous flow until such a point when their buoyant force which varies directly proportionate with their size is strong enough to overcome the surface tension forces, before finally breaking off. Under this low pressure state, the force difference is the key to bubble detachment from source and usually, bubbles are several orders of magnitude larger than their exit pore. Likewise, owing to coalescence between neighbouring bubbles, bubbles grow at least an order of magnitude bigger than the exit pore under steady air flow condition. This tendency nevertheless, is reduced under oscillatory air flow regime. The inertia of the pulse arising due to fludic oscillation overcomes the wetting force (see Hanly et al., 2011) directly, and with much less dissipation. Without oscillation, bubbles tend to move irregularly, leading to increased bubble-bubble interaction and consequently production of large bubbles. Regular detachment leads to less coalescence as the bubbles are more uniformly spaced and sized. The level of inertial force in the pulse can be tuned so that bubbles emerge with little excess kinetic energy over the terminal rise velocity (Parkinson et al. , 2008).

\subsection{Algal Recovery}

Understanding the step-wise processes prevalent in a multi-floc system between particle-bubble interaction in a flotation column is both interesting and informative. The photograph of the flotation unit illustrating key stages in the recovery process is shown in Figure 5. At first (Fig. 5a), the sludge blanket begins to form and sludge build 
up intensifies. Here, larger flocs are preferentially collected first before smaller flocs and the removal efficiency decreases sharply with the gradient of biomass with time (Fig 6).

This outcome is simply attributable to their large surfaces which readily render them susceptible to bubble collision and adhesion, bubble formation at particle surface, microbubble entrapment in aggregates and bubble entrainment by aggregates. (Edzwald, 2010) reported these bubble-particle interaction mechanisms in the review of flotation as a wastewater treatment. These large flocs also engage in sweep flocculation as they travel upwards under the lift of microbubbles hence the exponential biomass recovery efficiency recorded at the early stage.

After half the separation time (Stage 1), the amount of large flocs decreases markedly in the continuous phase; smaller flocs become prevalent in the flotation unit, indicating the second key stage. Biomass concentration (Fig. 6) only reduces slightly and as such recovery efficiency therefore increases fractionally with time because at fixed bubble size, bubble-particle contact is more effective with large flocs. In the second stage (Fig $5 b$ ), sludge build up continuous but also observed is the thickening of the sludge blanket. As more bubbles rise to the top, these bubbles compress the sludge layer from underneath, reducing the water content of the sludge.

Schulze (1992) provides some supportive theoretical and experimental insight as to why this is. Bubble-particle interaction upon approach has been found to occur either by attachment or driftage. While the former often results in collision and bubble surface deformation following the extension of the thin liquid film between bubble surface and particle to create a three point contact, the latter process occurs across bubble surface and only causes minimal surface deformation without extension of the liquid film (Nguyen et al., 1997). 
(a)

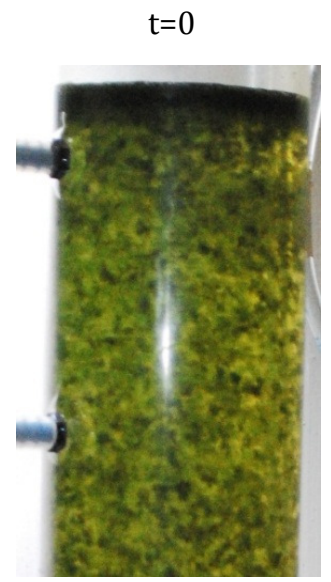

(b)

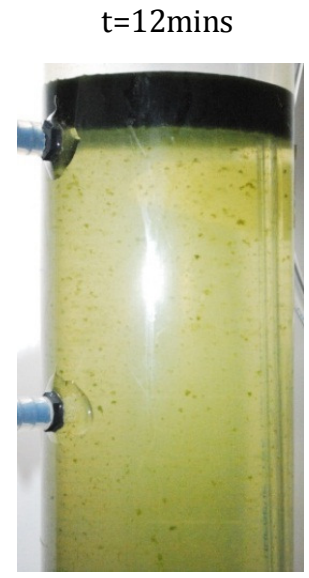

(c)

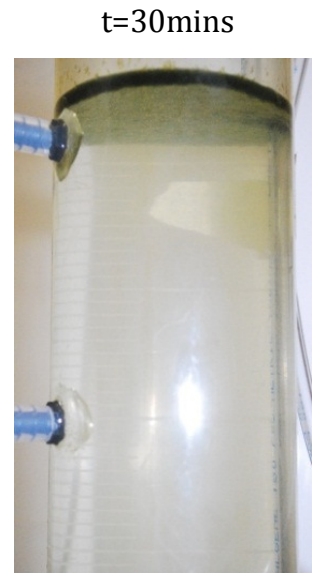

Figure 5: Photograph of the flotation unit showing the separation at three different key stages. (a) Few minutes after flocculated algal cells were introduced into the unit. Development of the sludge blanket outline begins to occur immediately as microbubbles transport large flocs. (b) Image of separated continuous phase clearly showing the algae sludge blanket minutes afterwards. Small flocs are predominant at this stage but the sludge layer is clearly outlined and fully formed (c) Third stage is marked by much slower separation as relatively smaller flocs but intense sludge thickening is observed. Clear continuous medium indicating full separation is obtained.

The possibility of particle-bubble collision in a flotation unit is higher with heavier and large particles at high radial particle velocity. By contrast, at relatively low velocities and with smaller particles, sliding (driftage) dominants the bubble particle contact mechanism (Schulze, 1992). And as such collision and attachment between particle and bubble is relatively low. Furthermore, the particle-bubble encounter probability and the collection probability are only equal when the collision as well as attachment probability is equal to 1 and the detachment probability, zero. This condition is rapidly obtainable given the two circumstances: particles are hydrophobic and sufficiently large for collision (Nguyen, 1998). The third key stage (Fig 5c) is primarily characterised by intensive sludge thickening and thinning. At this stage, the majority of the particles have been separated (Fig. 6) therefore microbubble rise velocity is increased as relatively very few particles are present to cause rise retardation and the rate of water removal from the sludge is high. The sludge layer is reduced to almost a quarter of the initial size. 


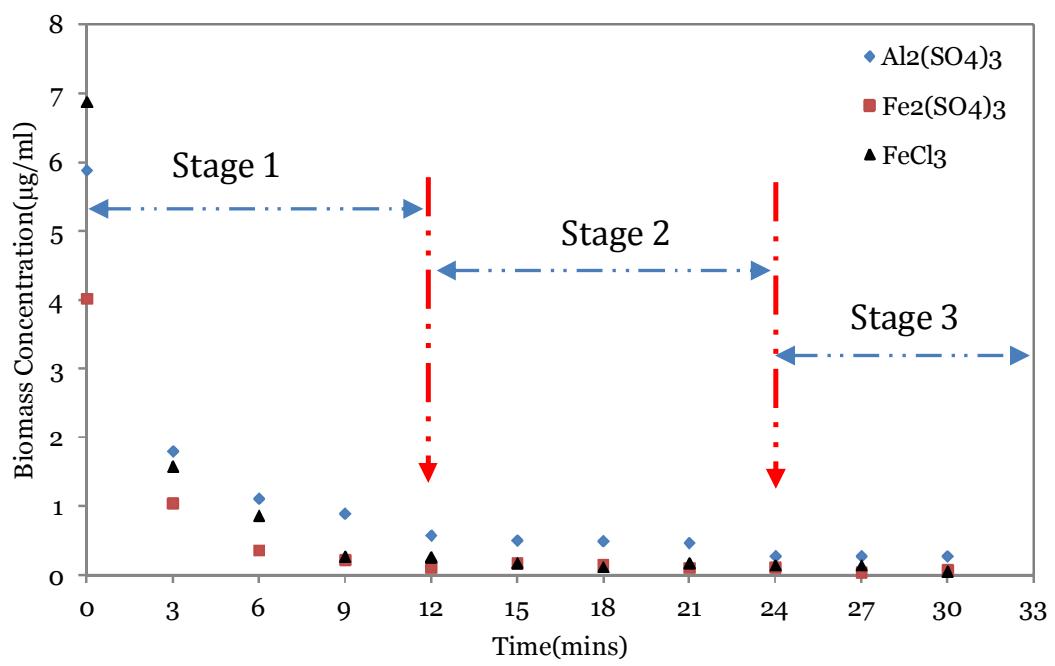

Figure 6: Graph of Algal biomass concentration as a function of time illustrating the different key stages in the flotation experiment. The first stage is characterised by sludge formation and intense sludge build-up, at this stage, separation efficiency is exponential and concentration of residual biomass drops sharply. Second stage still supports sludge build up but a transition into sludge thickening is observed. Here, separation efficiency is rather linear and biomass concentration reduces only gradually. Stage 3 is primarily dominated by sludge thickening and thinning, almost no significant separation efficiency result is recorded.

Table 1: Recovery efficiencies for the various sampling ports under best operating parameter (pH5 and $150 \mathrm{mg} / \mathrm{L}$ coagulant dose). Efficiencies of 94-99.2\% are reported across all ports and coagulant types. However, Teixeira and Rosa (2006) reported removal efficiencies of 92-98\% and 70-94\% for DAF and sedimentation respectively of blue-green algae while Wyatt et al., (2011) obtained 90\% removal efficiency of fresh water algae by flocculation.

\begin{tabular}{|c|c|c|c|c|}
\hline \multirow{2}{*}{$\begin{array}{c}\text { Sampling } \\
\text { Ports }\end{array}$} & \multirow{2}{*}{$\begin{array}{c}\text { Height } \\
\text { from }\end{array}$} & \multicolumn{3}{|c|}{ Coagulants } \\
\cline { 3 - 5 }$(\mathrm{SP})$ & Diffuser $(\mathrm{cm})$ & $\mathrm{Al}_{2}\left(\mathrm{SO}_{4}\right)_{3}$ & $\mathrm{Fe}_{2}\left(\mathrm{SO}_{4}\right)_{3}$ & $\mathrm{FeCl}_{3}$ \\
\cline { 3 - 5 } & 2 & 95.2 & 98.1 & 99.2 \\
\hline $\mathrm{Sp} 1$ & 10 & 94.2 & 98.3 & 98.9 \\
$\mathrm{Sp} 2$ & 18 & 94.6 & 98.6 & 98.9 \\
$\mathrm{Sp} 3$ & 26 & 93.9 & 96.9 & 98.2 \\
\hline $\mathrm{Sp} 4$ & & & & \\
\hline
\end{tabular}

\section{2 $\quad$ Effect of $\mathbf{p H}$}

Chemical pre-treatment is very essential in decreasing the effect of repulsive charge between bubbles and flocs. The success of chemical pre-treatment depends on $\mathrm{pH}$ because $\mathrm{pH}$ determines the solubility of chemical constituents of nutrient and metals in solution and influences the form and quantity of ions produced. Optimum $\mathrm{pH}$ and coagulant dosing reduces the charge on particles to about zero causing particles to be 
more hydrophobic (Edzwald, 2010). To investigate the effect of pH on separation, trials were conducted across different $\mathrm{pH}$ levels and results reported in Figure 6.

Figure 7 presents the flotation results for three metallic coagulants. The effect of $\mathrm{pH}$ on algal removal efficiency from Figure $7(a)$ showed that with aluminium sulphate coagulant, efficiency increases with decrease in $\mathrm{pH}$ to the lowest at $\mathrm{pH} 7$ before rising again as $\mathrm{pH}$ increases to 9 . Optimum recovery result of $95.2 \%$ was obtained at $\mathrm{pH} 5$ with efficiency gradually decreasing to $71.9 \%$ at $\mathrm{pH} 6$ and $50.6 \%$ at $\mathrm{pH} 7$. At $\mathrm{pH} 8$ however, a sudden increase to $74.6 \%$ was obtained and $81.5 \%$ at $\mathrm{pH} 9$ indicating the other peak of result with aluminium sulphate. Data from Figure 7(b) can be compared with the data in Fig.7 (a) which showed a similar trend in the effect of pH on algal recovery efficiency. Again two peaks were observed on either side of the $\mathrm{pH}$ range experimented in this study. Best results were obtained at pH 5 with $98.1 \%$ followed by $91.6 \%$ at $\mathrm{pH} 6$. The drop in performance continued to $83.2 \%$ at pH 7 before hitting the lowest with $80 \%$ at pH 8. At pH 9 however, the performance was observed to rise sharply to 85.5\%. From the result in Figure $7(\mathrm{c})$, it is apparent that the result with this coagulant was different. Algal recovery efficiency dropped monotonically and nearly linearly with $\mathrm{pH}$ decrease. Optimum result of $99.2 \%$ was achieved at $\mathrm{pH} 5$ and then $93.1 \%$ at $\mathrm{pH}$. The recovery result further decreased to $90 \%$ for both $\mathrm{pH} 7$ and pH 8 respectively and finally to $86.4 \%$ at $\mathrm{pH}$ 9. Graph 7 (c) is quite revealing in several ways. First, unlike the first two graphs, overall efficiency was higher. The least efficiency at pH 9 was higher than the $80 \%$ mark. Thus with this coagulant, efficiency ranged from 86.4\%-99.2\%.

(a)

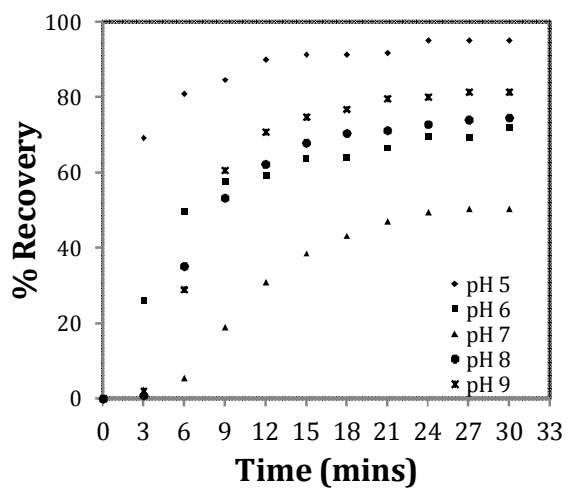

(b)

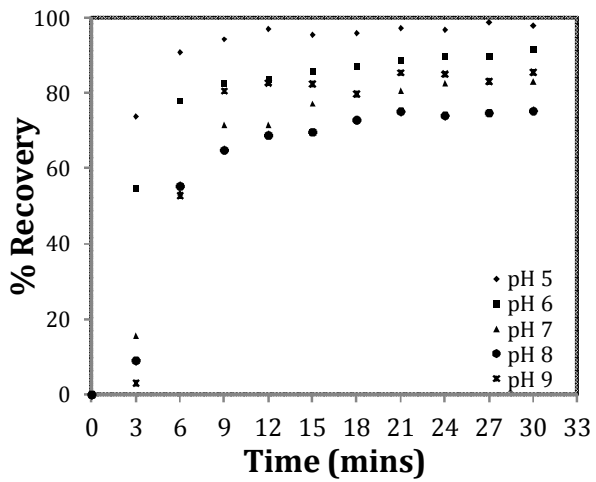

(c)

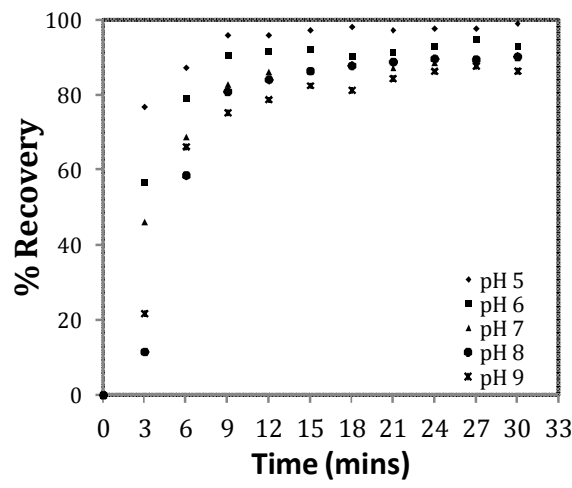

Figure 7: Graph of recovery efficiency at $150 \mathrm{mg} / \mathrm{L}$ coagulant dose against time at varying $\mathrm{pH}$ levels for all three metallic coagulants. Recovery efficiency for all three coagulant used is highest at pH 5. Under this condition however, Ferric Chloride gave overall best result followed by Ferric Sulphate then Aluminium Sulphate. 
In general, the optimum cell recovery result in these experiments was found at the lowest pH studied. One explanation for the non-monotonic behaviour observed for ferric sulphate is contactless flotation (Jiang et al. 2010). One would infer that isoelectric points for all three coagulants are achieved with acidic conditions, so the alkaline high separation with ferric chloride would not naturally be achieved by zeta potential neutrality. By adding metallic inorganic coagulants such as iron and aluminium salts in solution, coagulation is achieved with the coagulants dissociating into $\mathrm{Fe}^{3+}$ and $\mathrm{Al}^{3+}$ respectively as well as other soluble complexes having varying high positive charges. Essentially, the rate and extent to which these trivalent ions and other complexing species adsorb onto colloidal surfaces is $\mathrm{pH}$ dependent. At room temperature, under acidic $\mathrm{pH}$, trivalent species-Fe ${ }^{3+}$ (Wyatt et al., 2011) and $\mathrm{Al}^{3+}$ (Pernitsky and Edzwald, 2006) are the dominant species in the continuous phase These predominant trivalent species are the most effective in colloidal charge neutralization and attach to the negatively charged algal cell. The excess $\mathrm{H}^{+}$present under low $\mathrm{pH}$ react with hydroxides of these metals to further release the trivalent metal species. As a consequence, more $\mathrm{Al}^{3+}$ and $\mathrm{Fe}^{3+}$ species become available again for charge neutralization but the amount of hydroxides species is reduced. As $\mathrm{pH}$ shifts away from acidity however, $\mathrm{H}^{+}$concentration becomes less than $\mathrm{OH}^{-}$and the amount of trivalent ions present in solution reduces. These prevalent $\mathrm{OH}^{-}$react freely with the available trivalent metallic species to form the corresponding metallic hydroxide species. As such, hydroxide species become predominant under alkaline conditions attaching to algal cells and precipitating as large gelatinous flocs. Pernitsky and Edzwald, (2006) and Wyatt et al. (2011) reported increased concentrations of hydroxide species for aluminium and ferric salts respectively as $\mathrm{pH}$ moves beyond $\mathrm{pH} 7$ at room temperature. This explains the large flocs generated under alkaline condition. It is for these reasons the recovery efficiency is observed to increase again under alkaline $\mathrm{pH}$.

Considering that large flocs are good vehicles for sweep flocculation, one might wonder why, despite the relatively large flocs formed at pH greater than 7 , the overall efficiency under alkaline conditions recorded for aluminium and ferric sulphate coagulants was still lower than results under acidic state. Under the same operating conditions of flowrate, bubble size and flux, this observation can be explained by the difference in charge density of species. The higher the size and charge of the species, the more 
effective the coagulation process will be. Because these charges increase with increasing acidity, recovery efficiency is highest under acidic $\mathrm{pH}$. In addition, relatively larger flocs are developed under alkaline state and given that as particle size increases the residence time of the rising microbubble-floc agglomerate also increases leading to prolonged flotation time. Moreover, the lifting force of microbubbles diminishes with increased particle size (Miettinen et al., 2010).

By contrast, the condition is quite different for $\mathrm{FeCl}_{3}$ though. Whilst a similar tendency occurs under acidic condition, $\mathrm{FeCl}_{3}$ exhibits a rather different behaviour under basic $\mathrm{pH}$. It is note-worthy to reiterate that ferric chloride produced the overall best recovery result. The justification for this is that ferric salts are relatively less soluble than aluminium salts. This observation corresponds with the findings of Chow et al. (1998) on the concentration of iron speciation in solution. Their results showed that the soluble ion concentrations were less than $1 \%$ of the total iron chloride amount initially added. In addition, hydroxides of aluminium are amphoteric- containing both basic and acidic functional groups. Furthermore, the addition of ferric salts decreases the solution $\mathrm{pH}$ and the closer the $\mathrm{pH}$ tends towards acidity, concentration of trivalent species in the solution increases. Wyatt et al. (2011) observed the same occurrence in their study of critical conditions for ferric chloride-induced flocculation of freshwater algae The optimum $\mathrm{pH}$ for algal separation ranges from 5-7 for ferric chloride but for aluminium and ferric sulphate, two ranges are effective- 5-6 and 8-9. Overall, the process governing these reactions is very complex and by no means easy to fully detail especially also as the growth medium contains vital and very reactive chemical constituents.

\subsection{Effect of Coagulant Dose}

To ensure charge neutralization and proper particle agglomeration, good coagulation not only involves the type of coagulant but also the right amount of coagulant. By neutralising particle charge, collision between particles and bubbles is proliferated. The effect of coagulant concentration on separation efficiency was studied. Figure 8 shows the results of the effects of coagulant dose with time for the three metallic coagulants used.

A steady drop in efficiency was recorded with aluminium sulphate as the concentration of coagulant reduced from $150 \mathrm{mg} / \mathrm{L}$ to $25 \mathrm{mg} / \mathrm{L}$. Highest result obtained was $95 \%$ at $150 \mathrm{mg} / \mathrm{L}$. At $100 \mathrm{mg} / \mathrm{L}, 89.7 \%$ efficiency was obtained followed by $87.9 \%$ at $50 \mathrm{mg} / \mathrm{L}$ 
and then $80.5 \%$ for $75 \mathrm{mg} / \mathrm{L}$ before recording the lowest - $60.1 \%$ - at $25 \mathrm{mg} / \mathrm{L}$. With ferric sulphate, lowest yield in recovery efficiency was recorded at $25 \mathrm{mg} / \mathrm{L}$ which gave a maximum of $72.8 \%$ algal recovery followed by $83 \%, 86.8 \%$ and $92.7 \%$ for $50 \mathrm{mg} / \mathrm{L}$, $75 \mathrm{mg} / \mathrm{L}$ and $100 \mathrm{mg} / \mathrm{L}$ respectively. However, best algal recovery result recorded for this coagulant was achieved at $150 \mathrm{mg} / \mathrm{L}$ with a recovery efficiency of $98.1 \%$. The same exponential trend is observed with ferric chloride. Under this condition however, the lowest results registered were $86.4 \%$ and $93.9 \%$ at $25 \mathrm{mg} / \mathrm{L}$ and $50 \mathrm{mg} / \mathrm{L}$ respectively then, at $75 \mathrm{mg} / \mathrm{L}$ of coagulant dose, the results rose to $98.7 \%$ and then to $98.9 \%$ at $100 \mathrm{mg} / \mathrm{L}$. For $150 \mathrm{mg} / \mathrm{L}$ however, overall recovery efficiency of algal biomass obtained was $99.2 \%$.

(a)

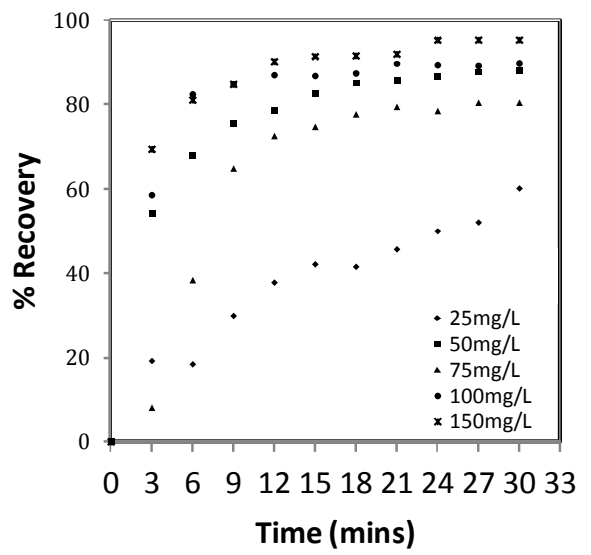

(b)

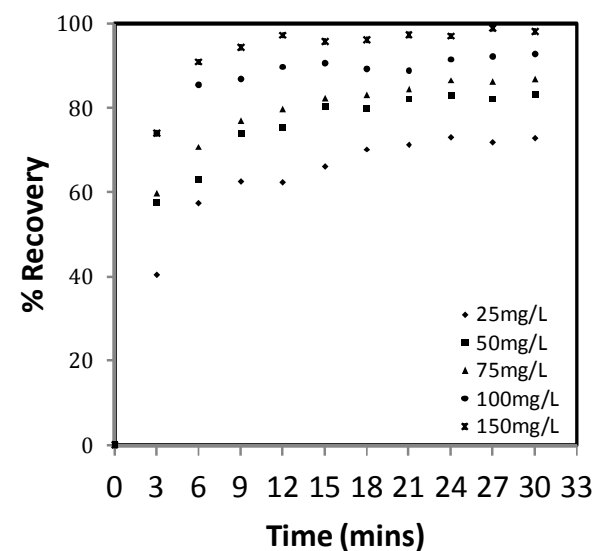

(c)

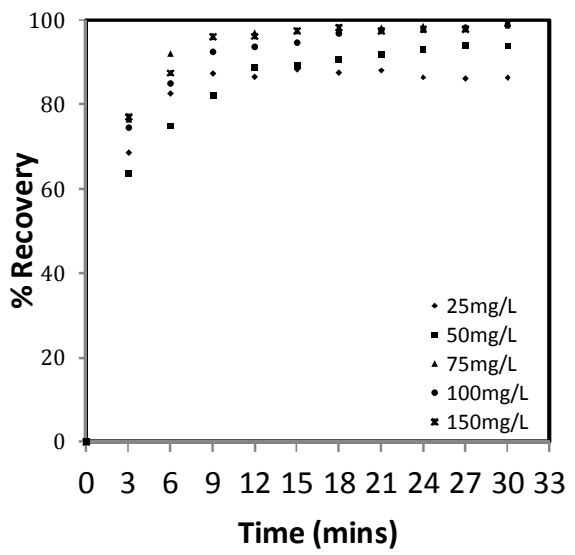

Figure 8: A plot of Algae recovery efficiency at $\mathrm{pH} 5$ as a function of time at varying coagulant concentrations for the three metallic coagulant types. A steady increase in algal cell recovery was recorded with increasing concentration of coagulant. For all three coagulants, highest result was obtained at $150 \mathrm{mg} / \mathrm{L}$ coagulant dose whilst the lowest results were recorded for $50 \mathrm{mg} / \mathrm{L}$ and $25 \mathrm{mg} / \mathrm{L}$ respectively.

The graphs in Figure 8 above reveal in all cases an increase in the recovery of algal cells as concentration of coagulant increases. This is so because compression of the double layer effect is essential for particles to agglomerate and within the isoelectric point, increasing the dosage of coagulant, provides more trivalent ions necessary for double layer compression. Bubble particle attachment and detachment in flotation studies by (Ralston et al., 1999) rightly supports this observation. The authors reported that increasing the amount of electrolytes decreases the interaction potential energy existing between bubble and particle. This phenomenon is more effective with hydrophobic 
particles. Also, when electrolyte concentration increases and at high particle hydrophobic strength, attachment efficiency becomes less dependent on size of particle.

The ability of a chemical coagulant to produce good coagulation is reliant on both the electric charge of the species and the size of the species used as coagulant. The higher the size and charge of the species, the more effective the coagulation process will be. Because these charges increase with increasing acidity, recovery efficiency increases at low pH. Coagulation of effluent is the most vital operating control variable influencing the performance of flotation. At low or no coagulation, particles remain negatively charged and hydrophilic which is why bubble-particle attachment is low or zero.

\subsection{Conclusion}

This study considered the performance of microflotation on algal biomass recovery. There are 4 (four) conclusions that can be drawn from the results. First, the fluidic oscillator generated bubbles about twice the size of their outlet pores. Second, fluidic oscillator generated microbubbles were effective in the recovery of algal biomass from growth medium. Third, algal biomass recovery was enhanced with increasing coagulant dose. Fourth, the effect of $\mathrm{pH}$ was a key factor in flocculation and recovery efficiency was optimum under acidic condition.

Good coagulation chemistry relies on coagulation $\mathrm{pH}$ and coagulant concentration. Best coagulation conditions for bubble-particle capture efficiency is a balance between appropriate $\mathrm{pH}$ and coagulant dose to generate flocs with reduced surface charge and high hydrophobicity. Optimum results was obtained at lowest $\mathrm{pH}$ for all three metal coagulant used. However, recovery efficiency showed exponential response with increasing coagulant dose.

As interest in sustainable energy continues to intensify, developing an energy efficient harvesting technique has never been more important. With the high energy cost associated with dissolved air flotation and the inefficiency of conventional dispersed air flotation to generate the right size of microbubbles, microflotation facilitated by fluidic oscillation is a viable technology that promises to meet both the generation of microbubbles and its application in water treatment or algal biomass recovery for biofuel production. 


\section{Acknowledgements}

WZ would like to acknowledge support from the Concept Fund of Yorkshire Forward and the EPSRC (grant no. EP/I019790/1). WZ would like to acknowledge the Royal Society for a Brian Mercer Innovation award and the Royal Academy of Engineering for an industrial secondment with AECOM Design Build (Brenda Franklin). JOH would like to thank the University of Sheffield for a doctoral scholarship. Many thanks also to Vaclav Tesar and Buddhi Hewakandamby for helpful discussions as well as Omotoun Shada and Kelly Murphy for assistance.

\subsection{References}

AL-SHAMRANI, A. A., JAMES, A. \& XIAO, H. 2002a. Destabilisation of oil-water emulsions and separation by dissolved air flotation. Water Research, 36, 1503-1512.

AL-SHAMRANI, A. A., JAMES, A. \& XIAO, H. 2002b. Separation of oil from water by dissolved air flotation. Colloids and Surfaces A: Physicochemical and Engineering Aspects, 209, 15-26.

BOROWITZKA, M. 1992. Algal biotechnology products and processes - matching science and economics. Journal of Applied Phycology, 4, 267-279.

CHISTI, Y. 2007. Biodiesel from microalgae. Biotechnology Advances, 25, 294-306.

DAI, Z., DUKHIN, S., FORNASIERO, D. \& RALSTON, J. 1998. The Inertial Hydrodynamic Interaction of Particles and Rising Bubbles with Mobile Surfaces. Journal of Colloid and Interface Science, 197, 275-292.

DAI, Z., FORNASIERO, D. \& RALSTON, J. 2000. Particle-bubble collision models -- a review. Advances in Colloid and Interface Science, 85, 231-256.

DERJAGUIN, B. V. \& DUKHIN, S. S. 1993. Theory of flotation of small and medium-size particles. Progress in Surface Science, 43, 241-266.

EDZWALD, J. K. 1995. Principles and applications of dissolved air flotation. Water Science and Technology, 31, 1-23.

EDZWALD, J. K. 2010. Dissolved air flotation and me. Water Research, 44, 2077-2106

EL-KAYAR, A., HUSSEIN, M., ZATOUT, A. A., HOSNY, A. Y. \& AMER, A. A. 1993. Removal of oil from stable oil-water emulsion by induced air flotation technique. Separations Technology, 3, 2531.

ENGLERT, A. H., RODRIGUES, R. T. \& RUBIO, J. 2009. Dissolved air flotation (DAF) of fine quartz particles using an amine as collector. International Journal of Mineral Processing, 90, 27-34.

GOCHIN, R. J. \& SOLARI, J. 1983. The role of hydrophobicity in dissolved air flotation. Water Research, 17, 651-657.

HOSNY, A. Y. 1996. Separating oil from oil-water emulsions by electroflotation technique. Separations Technology, 6, 9-17.

KITCHENER, J. A. \& GOCHIN, R. J. 1981. The mechanism of dissolved air flotation for potable water: basic analysis and a proposal. Water Research, 15, 585-590.

LI, X.-B., LIU, J.-T., WANG, Y.-T., WANG, C.-Y. \& ZHOU, X.-H. 2007. Separation of Oil from Wastewater by Column Flotation. Journal of China University of Mining and Technology, 17, 546-551, 577.

MIETTINEN, T., RALSTON, J. \& FORNASIERO, D. 2010. The limits of fine particle flotation. Minerals Engineering, 23, 420-437.

NGUYEN, A. V. 1998. Particle-bubble encounter probability with mobile bubble surfaces. International Journal of Mineral Processing, 55, 73-86.

NGUYEN, A. V., SCHULZE, H. J. \& RALSTON, J. 1997. Elementary steps in particle-bubble attachment. International Journal of Mineral Processing, 51, 183-195. 
PERNITSKY, D. J. \& EDZWALD, J. K. 2006. Selection of alum and polyaluminum coagulants: principles and applications.

RALSTON, J., FORNASIERO, D. \& HAYES, R. 1999. Bubble-particle attachment and detachment in flotation. International Journal of Mineral Processing, 56, 133-164.

SCHULZE, H. J. 1992. Probability of particle attachment on gas bubbles by sliding. Advances in Colloid and Interface Science, 40, 283-305.

TEIXEIRA, M. R. \& ROSA, M. J. 2006. Comparing dissolved air flotation and conventional sedimentation to remove cyanobacterial cells of Microcystis aeruginosa Part 1: The key operating conditions. Separation and Purification Technology, 52, 84-94.

TEIXEIRA, M. R., SOUSA, V. \& ROSA, M. J. 2010. Investigating dissolved air flotation performance with cyanobacterial cells and filaments. Water Research, 44, 3337-3344.

TESAŘ, V. \& BANDALUSENA, H. 2011. Bistable diverter valve in microfluidics. Experiments in Fluids, $50,1225-1233$.

WU, X.-J. \& CHAHINE, G. L. 2010. Development of an acoustic instrument for bubble size distribution measurement. Journal of Hydrodynamics, Ser. B, 22, 330-336.

WYATT, N. B., GLOE, L. M., BRADY, P. V., HEWSON, J. C., GRILLET, A. M., HANKINS, M. G. \& POHL, P. I. 2011. Critical conditions for ferric chloride-induced flocculation of freshwater algae. Biotechnology and Bioengineering, n/a-n/a.

ZIMMERMAN, W. B., HEWAKANDAMBY, B. N., TESAR, V., BANDULASENA, H. C. H. \& OMOTOWA, O. A. 2009. On the design and simulation of an airlift loop bioreactor with microbubble generation by fluidic oscillation. Food and Bioproducts Processing, 87, 215-227.

ZIMMERMAN, W. B., TESAR, V. \& BANDULASENA, H. C. H. 2011a. Towards energy efficient nanobubble generation with fluidic oscillation. Current Opinion in Colloid \& Interface Science, In Press, Corrected Proof.

ZIMMERMAN, W. B., TESAŘ, V., BUTLER, S. \& BANDULASENA, H. 2008. Microbubble Generation. Recent Patents on Engineering, 2, 1-8.

ZIMMERMAN, W. B., ZANDI, M., HEMAKA BANDULASENA, H. C., TESAR, V., JAMES GILMOUR, D. \& YING, K. 2011b. Design of an airlift loop bioreactor and pilot scales studies with fluidic oscillator induced microbubbles for growth of a microalgae Dunaliella salina. Applied Energy, In Press, Corrected Proof.

ZIMMERMAN, W. B., ZANDI, M., HEMAKA BANDULASENA, H. C., TESAR, V., JAMES GILMOUR, D. \& YING, K. 2011c. Design of an airlift loop bioreactor and pilot scales studies with fluidic oscillator induced microbubbles for growth of a microalgae Dunaliella salina. Applied Energy, 88, 3357-3369.

ZOUBOULIS, A. I. \& AVRANAS, A. 2000. Treatment of oil-in-water emulsions by coagulation and dissolved-air flotation. Colloids and Surfaces A: Physicochemical and Engineering Aspects, $172,153-161$. 